\title{
Supplementary material for: Sparse oracle inequalities for variable selection via regularized quantization
}

Clément Levrard

\section{Proof of Proposition 2.3}

A bounded difference inequality such as Theorem 6.2 of [?] yields, for $x>0$,

$$
\mathbb{P}\left(\max _{p=1, \ldots, d}\left|\frac{\hat{\sigma}_{p}^{2}}{\sigma_{p}^{2}}-1\right| \geq \mathbb{E}\left[\max _{p=1, \ldots, d}\left|\frac{\hat{\sigma}_{p}^{2}}{\sigma_{p}^{2}}-1\right|\right]+x\right) \leq e^{-\frac{2 n x^{2}}{T^{4}(\sigma)}} .
$$

Besides, Theorem 2.8 of [?] ensures that, for every $p=1, \ldots, d, \hat{\sigma}_{p}^{2} / \sigma_{p}^{2}-1$ is a subgaussian random variable with variance bounded by $T(\sigma)^{4} / 4 n$. For a comprehensive introduction to subgaussian random variables and its application to empirical process theory, the interested reader is referred to Section 2.3 of [?] or Chapter 14 of [?]. A direct application of Theorem 2.5 of [?] leads to

$$
\mathbb{E}\left[\max _{p=1, \ldots, d}\left|\frac{\hat{\sigma}_{p}^{2}}{\sigma_{p}^{2}}-1\right|\right] \leq \frac{T(\sigma)^{2} \sqrt{\log (d)}}{\sqrt{2 n}}
$$

hence

$$
\mathbb{P}\left(\max _{p=1, \ldots, d}\left|\frac{\hat{\sigma}_{p}^{2}}{\sigma_{p}^{2}}-1\right| \geq \frac{T(\sigma)^{2} \sqrt{\log (d)}}{\sqrt{2 n}}\left[1+\sqrt{\frac{x}{\log (d)}}\right]\right) \leq e^{-x}
$$

Since $\sqrt{2 n} \kappa_{1}>T(\sigma)^{2} \sqrt{\log (d)}$, choosing $x=\log (d)\left(\frac{\sqrt{2 n} \kappa_{1}}{T(\sigma)^{2} \sqrt{\log (d)}}-1\right)^{2}$ leads to the result.

For the threshold Lasso with unique optimal codebook, Theorem 3.1 in [?], combined with Assumption 2, provides a constant $C_{0}$ such that

$$
\left\|\hat{\mathbf{c}}_{n}-\mathbf{c}^{*}\right\| \leq C_{0} M \sqrt{\frac{k}{n}\left(1+\frac{x}{k}\right)},
$$

with probability larger than $1-e^{-x}$. Since, for every $p$ in $\{1, \ldots, d\}$,

$$
\left|\frac{\left\|\mathbf{c}^{*,(p)}\right\| \vee \delta}{\left\|\hat{\mathbf{c}}_{n}^{(p)}\right\| \vee \delta}-1\right| \leq \frac{\left|\left\|\mathbf{c}^{*,(p)}\right\| \vee \delta-\left\|\hat{\mathbf{c}}_{n}^{(p)}\right\| \vee \delta\right|}{\delta} \leq \frac{\left\|\hat{\mathbf{c}}_{n}-\mathbf{c}^{*}\right\|}{\delta}
$$

the results easily follows. 


\section{Proof of Proposition 4.1}

For a fixed $\mathbf{c}$ in $\mathbf{c}^{k}$, denote by $Z_{r}(\mathbf{c})$ the following random variable

$$
Z_{r}(\mathbf{c})=\sup _{I_{w}\left(\mathbf{c}^{\prime}-\mathbf{c}\right) \leq r}\left|\left(P-P_{n}\right)\left(\bar{\gamma}\left(\mathbf{c}^{\prime}, .\right)-\bar{\gamma}(\mathbf{c}, .)\right)\right|
$$

The following proposition gives a non-asymptotic bound on $Z_{r}(\mathbf{c})$.

Proposition B.1. Suppose that $k d>1$ and $w$ is deterministic. Let $x>0$, and $\mathbf{c}$ be a fixed codebook. Then, with probability larger than $1-e^{-x}$,

$$
Z_{r}(\mathbf{c}) \leq 8 \sqrt{2 \pi} \sqrt{\frac{k \log (k d)}{n}} r T(w)\left(1+\frac{1}{4 \sqrt{\pi}} \sqrt{\frac{x}{k \log (k d)}}\right) .
$$

The proof of Proposition B.1 is postponed to the next subsection. Proposition 4.1 derives from a peeling argument, as in Section 3.4 of [?], combined with Proposition B.1. Let $a$ be the smallest integer such that $e^{-(a-1)} 2 \bar{M}(w) \leq \lambda_{0}$, and take $u_{0}=\log (a)$ (we recall here that $\bar{M}(w)=\sqrt{k}\|w\|^{2} T(w)$ is an upper bound on $I_{w}(\mathbf{c})$, for $\mathbf{c}$ in $\left.C^{k}\right)$. Then it is easy to see that $u_{0} \leq u$, where we recall that $u$ is defined in Proposition 4.1 as $\log \left(\frac{\|w\|^{2} \sqrt{n}}{\sqrt{\log (k d}}\right)$. We may write

$$
\begin{aligned}
& \mathbb{P}\left(\sup _{I_{w}\left(\mathbf{c}^{\prime}-\mathbf{c}\right) \leq 2 \bar{M}(w)} \frac{\left|\left(P-P_{n}\right)\left(\bar{\gamma}\left(\mathbf{c}^{\prime}, .\right)-\bar{\gamma}(\mathbf{c}, .)\right)\right|}{I_{w}\left(\mathbf{c}^{\prime}-\mathbf{c}\right) \vee \lambda_{0}} \geq \lambda_{1}\right) \\
& \leq \sum_{j=1}^{a-1} \mathbb{P}\left(\sup _{\substack{I_{w}\left(\mathbf{c}^{\prime}-\mathbf{c}\right) \leq 2 e^{-(j-1)} \bar{M}(w) \\
I_{w}\left(\mathbf{c}^{\prime}-\mathbf{c}\right) \geq 2 e^{-j} \bar{M}(w)}} \frac{\left|\left(P-P_{n}\right)\left(\bar{\gamma}\left(\mathbf{c}^{\prime}, .\right)-\bar{\gamma}(\mathbf{c}, .)\right)\right|}{2 e^{-j} \bar{M}(w)} \geq \lambda_{1}\right) \\
& +\mathbb{P}\left(\sup _{I_{w}\left(\mathbf{c}^{\prime}-\mathbf{c}\right) \leq 2 e^{-(a-1)} \bar{M}(w)} \frac{\left|\left(P-P_{n}\right)\left(\bar{\gamma}\left(\mathbf{c}^{\prime}, .\right)-\bar{\gamma}(\mathbf{c}, .)\right)\right|}{2 e^{-a} \bar{M}(w)} \geq \lambda_{1}\right) \\
& \leq \sum_{j=1}^{a} \mathbb{P}\left(Z_{2 e^{-(j-1)}} \bar{M}(w) \geq 2 e^{-(j-1)} \bar{M}(w) \lambda_{0}\left(1+\sqrt{\frac{u+x}{k \log k d}}\right)\right) \\
& \leq a e^{-u} e^{-x} \text {, }
\end{aligned}
$$

where the last inequality follows from Proposition B.1. Noticing that $a e^{-u} \leq 1$ proves the result.

\section{Proof of Proposition B.1}

This proof is a slight modification of the proof of Theorem 3.1 in [?], and mainly relies on the use of Gaussian complexities combined with Slepian's Lemma (see, e.g., Theorem 
13.3 in [?]). For every $j=1, \ldots, k$, if $I_{w}\left(\mathbf{c}^{\prime}-\mathbf{c}\right) \leq r$, then, for all $x$ in $C$, using $\left|a^{2}-b^{2}\right| \leq$ $2 \max (|a|,|b|)|a-b|$ for $a, b$ in $\mathbb{R}$ and the Cauchy-Schwarz inequality, we get

$$
\begin{aligned}
\left|-2\left\langle x, c_{j}^{\prime}\right\rangle+\left\|c_{j}^{\prime}\right\|^{2}+2\left\langle x, c_{j}\right\rangle-\left\|c_{j}\right\|^{2}\right| & \leq 2 \sum_{p=1}^{d}|x|_{p}\left|c_{j}^{\prime(p)}-c_{j}^{(p)}\right|+\sum_{p=1}^{d}\left|c_{j}^{\prime(p)^{2}}-c_{j}^{(p)^{2}}\right| \\
& \leq 4 \sum_{p=1}^{d} \frac{M_{p}}{w_{p}} w_{p} \sum_{l=1}^{k} \mathbb{1}(l=j)\left|c_{l}^{\prime(p)}-c_{l}^{(p)}\right| \\
& \leq 4 T(w) I_{w}\left(\mathbf{c}^{\prime}-\mathbf{c}\right) \leq 4 r T(w) .
\end{aligned}
$$

which leads to

$$
\left\|\bar{\gamma}\left(\mathbf{c}^{\prime}, .\right)-\bar{\gamma}(\mathbf{c}, .)\right\|_{\infty}:=\sup _{x \in C}\left\|\bar{\gamma}\left(\mathbf{c}^{\prime}, x\right)-\bar{\gamma}(\mathbf{c}, x)\right\| \leq 4 r T(w) .
$$

As a consequence, a bounded difference concentration inequality (see, e.g., Theorem 6.2 in [?]) yields, with probability larger than $1-e^{-x}$,

$$
Z_{r}(\mathbf{c}) \leq \mathbb{E} Z_{r}(\mathbf{c})+4 r T(w) \sqrt{\frac{2 x}{n}} .
$$

It remains to bound from above $\mathbb{E} Z_{r}(\mathbf{c})$. According to the symmetrization principle (see, e.g., Section 2.2 of [?]), introducing some independent Rademacher variables $\varepsilon_{i}$ and standard Gaussian variables $g_{i}$ (also independent of the $\varepsilon_{i}$ 's), we have

$$
\begin{aligned}
\mathbb{E} Z_{r}(\mathbf{c}) & \leq 2 \mathbb{E}_{X} \mathbb{E}_{\varepsilon} \sup _{I_{w}\left(\mathbf{c}^{\prime}-\mathbf{c}\right) \leq r} \frac{1}{n} \sum_{i=1}^{n} \varepsilon_{i}\left(\bar{\gamma}\left(\mathbf{c}^{\prime}, X_{i}\right)-\bar{\gamma}\left(\mathbf{c}, X_{i}\right)\right) \\
& =2 \sqrt{\frac{\pi}{2}} \mathbb{E}_{X} \mathbb{E}_{\varepsilon} \sup _{I_{w}\left(\mathbf{c}^{\prime}-\mathbf{c}\right) \leq r} \mathbb{E}_{g}\left[\frac{1}{n} \sum_{i=1}^{n} \varepsilon_{i}\left|g_{i}\right|\left(\bar{\gamma}\left(\mathbf{c}^{\prime}, X_{i}\right)-\bar{\gamma}\left(\mathbf{c}, X_{i}\right)\right)\right] \\
& \leq 2 \sqrt{\frac{\pi}{2}} \mathbb{E}_{X} \mathbb{E}_{g} \sup _{I_{w}\left(\mathbf{c}^{\prime}-\mathbf{c}\right) \leq r} \frac{1}{n} \sum_{i=1}^{n} g_{i}\left(\bar{\gamma}\left(\mathbf{c}^{\prime}, X_{i}\right)-\bar{\gamma}\left(\mathbf{c}, X_{i}\right)\right) .
\end{aligned}
$$

Let $\mathbf{c}$ and $X_{1}, \ldots, X_{n}$ be fixed, and define, for $\mathbf{c}^{\prime}$ such that $I_{w}\left(\mathbf{c}^{\prime}-\mathbf{c}\right) \leq r$ the Gaussian process

$$
Y_{\mathbf{c}^{\prime}}=\sum_{i=1}^{n} g_{i}\left(\bar{\gamma}\left(\mathbf{c}^{\prime}, X_{i}\right)-\bar{\gamma}\left(\mathbf{c}, X_{i}\right)\right)
$$

Since, for every codebooks $\mathbf{c}_{1}^{\prime}$ and $\mathbf{c}_{2}^{\prime}$,

$$
\left(\bar{\gamma}\left(\mathbf{c}_{1}^{\prime}, X_{i}\right)-\bar{\gamma}\left(\mathbf{c}_{2}^{\prime}, X_{i}\right)\right)^{2} \leq \max _{j=1, \ldots, k} 8\left\langle c_{1, j}^{\prime}-c_{2, j}^{\prime}, X_{i}\right\rangle^{2}+2\left(\left\|c_{1, j}^{\prime}\right\|^{2}-\|\left. c_{2, j}^{\prime}\right|^{2}\right)^{2},
$$

it is easy to see that

$$
\operatorname{Var}\left(Y_{\mathbf{c}_{1}^{\prime}}-Y_{\mathbf{c}_{2}^{\prime}}\right) \leq \sum_{i=1}^{n} \sum_{j=1}^{k} 8\left\langle c_{1, j}^{\prime}-c_{2, j}^{\prime}, X_{i}\right\rangle^{2}+2 n \sum_{j=1}^{k}\left(\left\|c_{1, j}^{\prime}\right\|^{2}-\left\|c_{2, j}^{\prime}\right\|^{2}\right)^{2} .
$$


To derive bounds on the Gaussian complexity defined above, the following comparison result between Gaussian processes is needed.

Theorem B.1 (Slepian's Lemma). Let $\mathcal{V}$ be a separable metric space, and $Y_{t}, N_{t}, t$ in $\mathcal{V}$, be some continuous centered real Gaussian processes. Assume that

$$
\forall t_{1}, t_{2} \in \mathcal{V} \quad \operatorname{Var}\left(Y_{t_{1}}-Y_{t_{2}}\right) \leq \operatorname{Var}\left(N_{t_{1}}-N_{t_{2}}\right),
$$

then

$$
\mathbb{E} \sup _{t \in \mathcal{V}} Y_{t} \leq \mathbb{E} \sup _{t \in \mathcal{V}} N_{t}
$$

Theorem B.1 is a straightforward extension of Theorem 13.3 in [?] to separable index sets. Denote by $\mathcal{V}$ the separable set of codebooks $\mathbf{c}^{\prime}$ in $C^{k}$ such that $I_{w}\left(\mathbf{c}^{\prime}-\mathbf{c}\right) \leq r$. Now introduce, for $\mathbf{c}^{\prime}$ such that $I_{w}\left(\mathbf{c}^{\prime}-\mathbf{c}\right) \leq r$, the following Gaussian process

$$
N_{\mathbf{c}^{\prime}}=2 \sqrt{2} \sum_{i=1}^{n} \sum_{j=1}^{k}\left\langle c_{j}^{\prime}-c_{j}, X_{i}\right\rangle \xi_{i, j}+\sqrt{2 n} \sum_{j=1}^{k}\left(\left\|c_{j}^{\prime}\right\|^{2}-\left\|c_{j}\right\|^{2}\right) \xi_{j}^{\prime},
$$

where the $\xi^{\prime}$ 's and $\xi^{\prime}$ 's are independent standard Gaussian random variables. Note that $\mathbf{c}^{\prime} \mapsto N_{\mathbf{c}^{\prime}}$ is continuous, and for all $\mathbf{c}_{1}^{\prime}$ and $\mathbf{c}_{2}^{\prime}$ in $\mathcal{V}, \operatorname{Var}\left(Y_{\mathbf{c}_{1}^{\prime}}-Y_{\mathbf{c}_{2}^{\prime}}\right) \leq \operatorname{Var}\left(N_{\mathbf{c}_{1}^{\prime}}-N_{\mathbf{c}_{2}^{\prime}}\right)$. Consequently, applying Theorem B.1 yields

$$
\mathbb{E}_{g} \sup _{I_{w}\left(\mathbf{c}^{\prime}-\mathbf{c}\right) \leq r} Y_{\mathbf{c}^{\prime}} \leq \mathbb{E}_{\xi, \xi^{\prime}} \sup _{I_{w}\left(\mathbf{c}^{\prime}-\mathbf{c}\right) \leq r} N_{\mathbf{c}^{\prime}}
$$

It follows that

$$
\begin{aligned}
\mathbb{E}_{\xi, \xi^{\prime}} \sup _{I_{w}\left(\mathbf{c}^{\prime}-\mathbf{c}\right) \leq r} N_{\mathbf{c}^{\prime}} \leq \mathbb{E}_{\xi} \sup _{I_{w}\left(\mathbf{c}^{\prime}-\mathbf{c}\right) \leq r} 2 \sqrt{2} \sum_{i=1}^{n} & \sum_{j=1}^{k}\left\langle c_{j}^{\prime}-c_{j}, X_{i}\right\rangle \xi_{i, j} \\
& +\mathbb{E}_{\xi^{\prime}} \sup _{I_{w}\left(\mathbf{c}^{\prime}-\mathbf{c}\right) \leq r} \sqrt{2 n} \sum_{j=1}^{k}\left(\left\|c_{j}^{\prime}\right\|^{2}-\left\|c_{j}\right\|^{2}\right) \xi_{j}^{\prime} .
\end{aligned}
$$

The first term of the right side can be bounded as follows.

$$
\begin{aligned}
& \mathbb{E}_{\xi} \sup _{I_{w}\left(\mathbf{c}^{\prime}-\mathbf{c}\right) \leq r} 2 \sqrt{2} \sum_{i=1}^{n} \sum_{j=1}^{k}\left\langle c_{j}^{\prime}-c_{j}, X_{i}\right\rangle \xi_{i, j} \\
& \leq 2 \sqrt{2} \mathbb{E}_{\xi} \sup _{I_{w}\left(\mathbf{c}^{\prime}-\mathbf{c}\right) \leq r} \sum_{j=1}^{k}\left\langle c_{j}^{\prime}-c_{j}, \sum_{i=1}^{n} \xi_{i, j} X_{i}\right\rangle \\
& \leq 2 \sqrt{2} \mathbb{E}_{\xi} \sup _{I_{w}\left(\mathbf{c}^{\prime}-\mathbf{c}\right) \leq r}\left(\sum_{j=1}^{k} \sum_{p=1}^{d} w_{p}\left|c_{j}^{\prime(p)}-c_{j}^{(p)}\right|\right) \max _{j, p}\left|\sum_{i=1}^{n} \frac{\xi_{i, j} X_{i}^{(p)}}{w_{p}}\right| \\
& \leq 2 \sqrt{2 k} r \mathbb{E}_{\xi} \max _{j=1, \ldots, k, p=1, \ldots, d}\left|\sum_{i=1}^{n} \frac{\xi_{i, j} X_{i}^{(p)}}{w_{p}}\right| .
\end{aligned}
$$


Note that, for every $(j, p)$, the random variable $\sum_{i=1}^{n} \frac{\xi_{i, j} X_{i}^{(p)}}{w_{p}}$ is Gaussian, with variance bounded by $n T^{2}(w)$. Consequently, applying Theorem 2.5 in [?] gives

$$
\mathbb{E}_{\xi} \max _{j=1, \ldots, k, p=1, \ldots, d}\left|\sum_{i=1}^{n} \frac{\xi_{i, j} X_{i}^{(p)}}{w_{p}}\right| \leq T(w) \sqrt{2 n \log (k d)} .
$$

In turn, the second term of the right side may be bounded by

$$
\begin{aligned}
\mathbb{E}_{\xi^{\prime}} \sup _{I_{w}\left(\mathbf{c}^{\prime}-\mathbf{c}\right) \leq r} & \sqrt{2 n} \sum_{j=1}^{k}\left(\left\|c_{j}^{\prime}\right\|^{2}-\left\|c_{j}\right\|^{2}\right) \xi_{j}^{\prime} \\
& \leq \sqrt{2 n} \mathbb{E}_{\xi^{\prime}} \sup _{I_{w}\left(\mathbf{c}^{\prime}-\mathbf{c}\right) \leq r} \sum_{j=1}^{k}\left(\sum_{p=1}^{d} w_{p}\left|c^{\prime}{ }_{j}^{(p)}-c_{j}^{(p)}\right| \frac{2 M_{p}}{w_{p}}\right)\left|\xi_{j}^{\prime}\right| \\
& \leq 2 \sqrt{2 n} T(w) \mathbb{E}_{\xi^{\prime}} \sup _{I_{w}\left(\mathbf{c}^{\prime}-\mathbf{c}\right) \leq r} I\left(\mathbf{c}^{\prime}-\mathbf{c}\right) \sqrt{\sum_{j=1}^{k} \xi_{j}^{\prime 2}} \\
& \leq 2 T(w) r \sqrt{2 n k} .
\end{aligned}
$$

Combining these two bounds leads to

$$
\mathbb{E} Z_{r}(\mathbf{c}) \leq 8 \sqrt{2 \pi} \sqrt{\frac{k \log (k d)}{n}} r T(w) .
$$

\section{Proof of Proposition 4.2}

For every $u$ in $\mathbb{R}^{k}$, let us denote by $Y_{u}$ the function $\left\langle u, G^{(j)}\left(., \mathbf{c}^{*}\right)\right\rangle$, so that $\|(P-$ $\left.P_{n}\right) G^{(j)}\left(., \mathbf{c}^{*}\right) \|=\sup _{\|u\| \leq 1}\left(P-P_{n}\right) Y_{u}:=Y$. Since, for every $u$ such that $\|u\| \leq 1$ and for every $x$ in $C,\left|Y_{u}(x)\right| \leq M_{j}$, a bounded difference inequality yields (see, e.g., Theorem 6.2 in [?]), with probability larger than $1-e^{-x}$,

$$
Y \leq \mathbb{E} Y+M_{j} \sqrt{\frac{2 x}{n}} .
$$

An upper bound on $\mathbb{E} Y$ may be derived the same way as in the proof of Proposition B.1: introducing some Rademacher random variables $\varepsilon_{i}, i=1, \ldots, n$, and using the symmetrization principle, we get

$$
\begin{aligned}
\mathbb{E} Y & \leq 2 \mathbb{E}_{X, \varepsilon} \sup _{\|u\| \leq 1}\left\langle u, \frac{1}{n} \sum_{i=1}^{n} \varepsilon_{i} G^{(j)}\left(X_{i}, \mathbf{c}^{*}\right)\right\rangle \\
& \leq \frac{2}{n} \sqrt{\mathbb{E}_{X, \varepsilon}\left\|\sum_{i=1}^{n} \varepsilon_{i} G^{(j)}\left(X_{i}, \mathbf{c}^{*}\right)\right\|^{2}} \\
& \leq \frac{2 M_{j}}{\sqrt{n}}
\end{aligned}
$$


according to Cauchy-Schwarz and Jensen's inequalities. Choosing $x=f(n)$ gives the first concentration inequality of Proposition 4.2.

Now consider the $\{0,1\}$-valued random variables $Y_{\mathbf{c}}$, indexed by $C^{k}$, defined by $Y_{\mathbf{c}}=$ $\mathbb{1}_{\cup_{i \neq p} W_{i}(\mathbf{c}) \cap W_{p}\left(\mathbf{c}^{*}\right)}$. According to [?], the sets $\left\{\bigcup_{i \neq p} W_{i}(\mathbf{c}) \cap W_{p}\left(\mathbf{c}^{*}\right) \mid \mathbf{c} \in C^{k}\right\}$ have finite VC-dimension, say $D$. Using the well-known Vapnik-Chervonenkis bound (see, e.g., [?]), combined with a bounded difference concentration inequality yields

$$
P_{n} Y_{\mathbf{c}} \leq P Y_{\mathbf{c}}+K \sqrt{\frac{D}{n}}\left(1+\sqrt{\frac{x}{D}}\right),
$$

with probability larger than $1-e^{-x}$, for every $\mathbf{c}$ in $C^{k}$, and for some absolute constant $K$. Choosing $x=f(n)$ provides the second inequality of Proposition 4.2.

\section{Proof of Lemma 4.2}

The proof of Lemma 4.2 follows from the Proof of Proposition 3.2 in [?]. To give an upper bound on $R(\mathbf{m})$, we may write

$$
\begin{aligned}
R(\mathbf{m}) & =\sum_{i=1}^{k} \frac{\theta_{i}}{(2 \pi)^{d / 2} N_{i} \sqrt{\left|\Sigma_{i}\right|}} \sum_{j=1}^{k} \int_{W_{j}(\mathbf{m})}\left\|x-m_{j}\right\|^{2} e^{-\frac{1}{2}\left(x-m_{i}\right)^{t} \Sigma_{i}^{-1}\left(x-m_{i}\right)} \mathbb{1}_{\mathcal{B}(0, M)}(x) d x \\
& \leq \sum_{i=1}^{k} \frac{\theta_{i}}{(1-\eta)(2 \pi)^{d / 2} \sqrt{\left|\Sigma_{i}\right|}} \int_{\mathbb{R}^{d}}\left\|x-m_{i}\right\|^{2} e^{-\frac{1}{2}\left(x-m_{i}\right)^{t} \Sigma_{i}^{-1}\left(x-m_{i}\right)} d x \\
& \leq \sum_{i=1}^{k} \frac{\theta_{i}}{(1-\eta)(2 \pi)^{d / 2}} \int_{\mathbb{R}^{d}}\left\|\sqrt{\Sigma_{i}} u\right\|^{2} e^{-\frac{1}{2}\|u\|^{2}} d u
\end{aligned}
$$

where $\sqrt{\Sigma_{i}}$ denotes the square root of the matrix $\Sigma_{i}$. Since $\Sigma_{i}$ has its largest eigenvalue bounded by $\sigma^{2}$, it follows that $\left\|\sqrt{\Sigma_{i}} u\right\|^{2} \leq \sigma^{2}\|u\|^{2}$, for every $u$ in $\mathbb{R}^{d}$. We deduce that

$$
\begin{aligned}
R(\mathbf{m}) & \leq \frac{\sigma^{2} k \theta_{\max }}{(2 \pi)^{d / 2}(1-\eta)} \int_{\mathbb{R}^{d}}\|u\|^{2} e^{-\frac{1}{2}\|u\|^{2}} d u \\
& \leq \frac{\sigma^{2} k \theta_{\max } d}{(1-\eta)}
\end{aligned}
$$


which proves (15). Now let $\mathbf{c}$ be a codebook, and let $i$ be such that $\left\|c_{i}-m_{j}\right\|>\tau \tilde{B}$, for every $j$ in $\{1, \ldots, k\}$, with $\tau<1 / 2$. Since $\mathcal{B}\left(m_{i}, \tau \tilde{B} / 2\right) \subset \mathcal{B}(0, M)$, we may write

$$
\begin{aligned}
R(\mathbf{c}) & >\int_{\mathcal{B}\left(m_{i}, \tau \tilde{B} / 2\right)}\left(\frac{\tau \tilde{B}}{2}\right)^{2} \frac{\theta_{i}}{(2 \pi)^{d / 2} \sqrt{\left|\Sigma_{i}\right|}} e^{-\frac{1}{2}\left(x-m_{i}\right)^{t} \Sigma_{i}^{-1}\left(x-m_{i}\right)} d x \\
& >\frac{\theta_{\min } \tau^{2} \tilde{B}^{2}}{4(2 \pi)^{d / 2}} \int_{{\sqrt{\Sigma_{i}}}^{-1} \mathcal{B}(0, \tau \tilde{B} / 2)} e^{-\frac{1}{2}\|u\|^{2}} d u \\
& >\frac{\theta_{\min } \tau^{2} \tilde{B}^{2}}{4(2 \pi)^{d / 2}} \int_{\mathcal{B}\left(0, \frac{\tau \tilde{B}}{2 \sigma}\right)} e^{-\frac{1}{2}\|u\|^{2}} d u
\end{aligned}
$$

Since, for every positive $r,[-r / \sqrt{d}, r / \sqrt{d}]^{d} \subset \mathcal{B}(0, r)$, and $\int_{r}^{\infty} e^{-r^{2} / 2} d r \leq e^{-r^{2} / 2} / r$, it follows that

$$
\begin{aligned}
R(\mathbf{c}) & >\frac{\theta_{\min } \tau^{2} \tilde{B}^{2}}{4(2 \pi)^{d / 2}}\left(2 \int_{0}^{\frac{\tau \tilde{B}}{2 \sigma \sqrt{d}}} e^{-\frac{r^{2}}{2}} d r\right)^{d} \\
& >\frac{\tau^{2} \tilde{B}^{2} \theta_{\min }}{4}\left(1-\frac{2 \sigma \sqrt{d}}{\sqrt{2 \pi} \tau \tilde{B}} e^{-\frac{\tau^{2} \tilde{B}^{2}}{4 d \sigma^{2}}}\right)^{d},
\end{aligned}
$$

which proves (16). At last, let $\tau^{\prime}$ be such that $2 \tau+\tau^{\prime}<\frac{1}{2}$, and let $y$ be in $N_{\mathbf{c}^{*}}\left(\tau^{\prime} \tilde{B}\right)$. Then, for every $i$ in $\{1, \ldots, k\}$, we have $\left\|m_{i}-y\right\| \geq\left[\frac{1}{2}-\left(2 \tau+\tau^{\prime}\right)\right] \tilde{B}$. Hence

$$
f(y) \leq \frac{k \theta_{\max }}{(2 \pi)^{d / 2}(1-\eta) \sigma_{-}^{d}} e^{-\frac{\left[\frac{1}{2}-\left(2 \tau+\tau^{\prime}\right)\right]^{2} \tilde{B}^{2}}{2 \sigma^{2}}} .
$$

Since $\sigma_{-} \geq c_{-} \sigma$ and $\lambda\left(N_{\mathbf{c}^{*}}(t)\right) \leq 2 t k M^{d-1} S_{d-1}$, straightforward calculation leads to (17). 